\title{
Lo personal es político: un análisis feminista de la experiencia cotidiana de cuidadoras informales de personas adultas en situación de dependencia, en Santiago de Chile ${ }^{1}$
}

\author{
O pessoal é politico: uma análise feminista da experiência cotidiana de cuidadoras \\ informais de pessoas adultas em situação da dependência, em Santiago de Chile
}

\section{The personal is political: a feminist analysis of the daily experience of informal female caregivers of adult people in dependence situation, in Santiago de Chile}

Débora Ester Grandón Valenzuela ${ }^{a}$ (D)

aDepartamento de Terapia Ocupacional y Ciencia de la Ocupación, Universidad de Chile, Santiago de Chile, Chile.

Cómo citar: Grandón Valenzuela, D. E. (2021). Lo personal es político: un análisis feminista de la experiencia cotidiana de cuidadoras informales de personas adultas en situación de dependencia, en Santiago de Chile. Cadernos Brasileiros de Terapia Ocupacional, 29 , e2161. https://doi.org/10.1590/2526-8910.ctoAO2161

\begin{abstract}
Resumen
Introducción: El artículo plantea, desde una perspectiva feminista, cómo la creciente crisis del cuidado, acentuada en Estados neoliberales, genera una absorción de esta problemática por las mujeres, especialmente cuando se trata de personas adultas en situación de dependencia. Objetivo: Describir la cotidianidad de cuidadoras informales de personas adultas en situación de dependencia, además de los efectos que este trabajo ha tenido en sus vidas. Método: Investigación cualitativa, enmarcada como crítica y feminista, que ha incluido la entrevista semiestructurada y observación participante de la cotidianidad de 7 cuidadoras informales en Santiago de Chile, entre 2018 y 2019. Resultados: Se identificó una compleja trama de actividades que componen la cotidianidad del trabajo de cuidados, que producen gran nivel de sobrecarga en las cuidadoras. Además, se evidenciaron efectos múltiples en sus cotidianidades, que incluyen los de tipo personal, familiar y económico. Condusiones: La sexista organización social del cuidado produce ocupaciones generizadas como efecto, evidenciando el carácter sociohistórico que genera cotidianidades precarizadas para las mujeres que asumen el trabajo de cuidado de personas adultas en situación de dependencia de modo informal, como problema político y no personal.
\end{abstract}

Palabras-dave: División del Trabajo Basado en el Género, Actividades Cotidianas, Personas con Discapacidad, Perspectiva de Género, Feminismo, Terapia Ocupacional

\begin{abstract}
$\underline{\text { Resumo }}$
Introduçáo: $\mathrm{O}$ artigo propóe, a partir de uma perspectiva feminista, como a crescente crise de cuidado, acentuada nos estados neoliberais, gera uma absorção desse problema pelas mulheres, principalmente quando se trata de adultos em situação de dependência. Objetivo: Descrever o cotidiano de cuidadoras informais de adultos dependentes, além das repercussóes deste trabalho em suas vidas. Método: Pesquisa qualitativa, classificada como crítica e feminista, que realizou entrevistas semiestruturadas e observaçáo participante do cotidiano de 7 cuidadoras informais em Santiago do Chile, entre 2018 e 2019. Resultados: Foi identificada uma complexa teia de atividades que compóe o cotidiano do trabalho de cuidado, que produz alto nível de sobrecarga para as cuidadoras. Além disso, foram evidenciados múltiplos efeitos em seu cotidiano, que incluem os de ordem pessoal, familiar e econômica. Condusóes: A sexista organizaçâo social do cuidado produz como efeito ocupaçôes generizadas, evidenciando o caráter histórico-social que gera cotidianidades precárias para
\end{abstract}

\footnotetext{
${ }^{1}$ Este artículo es una síntesis de la investigación titulada "Lo personal es político: experiencias de mujeres cuidadoras de personas adultas en situación de dependencia en Santiago de Chile” desarrollada entre 2018 y 2019 en Chile, para obtener el grado de Magíster en Estudios de Género y Cultura, de la Universidad de Chile.
} 
mulheres que realizam informalmente trabalhos de cuidado de adultos em situação de dependência, como um problema político e não pessoal.

Palavras-chave: Divisão do Trabalho Baseado no Gênero, Atividades Cotidianas, Pessoas com Deficiência, Perspectiva de Gênero, Feminismo, Terapia Ocupacional

\begin{abstract}
$\underline{\text { Abstract }}$
Introduction: The article raises, from a feminist perspective, how the growing crisis of care, accentuated in neoliberal states, generates an absorption of this problem by women, especially when it comes to dependent adults. Objective: To describe the daily life of informal caregivers of dependent adults, in addition to the effects that this work has had on their lives. Method: Qualitative research, framed as critical and feminist, which has included the semi-structured interview and participant observation of the daily life of 7 informal caregivers in Santiago de Chile, between 2018 and 2019. Results: A complex web of activities was identified that make up the daily life of care work, which produces a great level of overload on caregivers. In addition, multiple effects were evidenced in their daily lives, which include those of a personal, family, and economic nature. Conclusions: The sexist social organization of care produces gendered occupations as its effect, evidencing the socio-historical character that generates precarious daily lives for women who undertake care work for dependent adults in an informal way as a political and not personal problem.
\end{abstract}

Keywords: Gender-Based Division of Labor, Activities of Daily Living, Disabled Persons, Gender Perspective, Feminism, Occupational Therapy.

\title{
Introducción: El Cuidado como Problema Político
}

Asistimos, a nivel mundial, a una creciente crisis social respecto del cuidado, a partir de lo que teóricas de la economía feminista han denominado como el conflicto capital-vida, esto es, la incompatibilidad entre la sostenibilidad de la vida, con el modelo socioeconómico de capitalismo neoliberal vigente (Pérez, 2014). Cuestiones como el progresivo desmantelamiento de los Estados de bienestar a nivel global, la participación activa de las mujeres en los mercados laborales remunerados, la privatización y mercantilización de la oferta de cuidados, el aumento del envejecimiento poblacional y la prevalencia de situaciones de discapacidad han creado escenarios hostiles para el desarrollo de las actividades de cuidado, las cuales en su mayoría, siguen siendo asumidas por mujeres, reproduciendo así una sexista organización social del cuidado (Gonzálvez, 2018).

Según las Encuestas Nacionales del Uso del Tiempo (ENUT) desarrolladas en diversos países latinoamericanos por la Comisión Económica para América Latina y el Caribe (CEPAL), se muestra un patrón que evidencia que pese al aumento de la participación laboral femenina en el trabajo remunerado, no ha aumentado la participación masculina en el trabajo doméstico y de cuidados (Instituto Nacional de Estadísticas de Chile, 2016). Según la Comisión Económica para América Latina y el Caribe (2011) "la conciliación entre la vida laboral y familiar basada en la redistribución de las tareas de cuidado entre el Estado, el mercado y las familias sigue siendo el punto ciego de las políticas públicas de América Latina y el Caribe" (p. 29), asunto de pronóstico poco alentador, pues además se prevé que:

En las próximas décadas se agudizará el proceso de envejecimiento de la población de la región y, por lo tanto, se incrementará la carga de cuidado de las personas adultas mayores, los enfermos crónicos y las personas con alguna discapacidad, y aumentarán los costos de la atención de la salud y los sistemas de pensiones (Comisión Económica para América Latina y el Caribe, 2017, p. 19).

En el caso de Chile, esta crisis se ha ido intensificando, pues además de los cambios demográficos contemporáneos caracterizados por la disminución de las tasas de natalidad y el aumento de la esperanza de vida, persiste una rígida división sexual del trabajo en los hogares que asume los vacíos de cuidado que el Estado no garantiza (Durán, 2011; Batthyány, 2015; Fraser, 2016). Es importante señalar que las políticas públicas chilenas sufrieron un radical cambio de foco tras la dictadura cívico-militar a partir de 1973, en donde se fueron desmantelando los diversos avances que se habían tenido hacia un Estado de Bienestar, en pos de la instalación de un profundo modelo neoliberal que ha privatizado desde entonces una serie de derechos sociales básicos (Arriagada, 2004; Arriagada \& Todaro, 2012). La inclusión de políticas públicas orientadas al cuidado de personas adultas que se encuentran en situaciones de dependencia —que es el foco de la presente investigación—, resulta sumamente reciente y con muy poco alcance a nivel nacional. El año 2016 se creó Chile Cuida, sistema de apoyos y cuidados del país, incluido en el sistema 
de protección social (Chile Cuida, 2020). Éste ofrece un programa de apoyo a los cuidados de personas en situación de dependencia, especialmente dirigido a adultos y adultas mayores, personas en situación de discapacidad y sus cuidadoras. Sin embargo, este programa tiene una serie de barreras de acceso, a saber: (1) sólo se desarrolla en 21 comunas $^{2}$ del país, lo que significa un 6.06\% con relación al total nacional (346 comunas); (2) sólo está dirigido al 60\% de los hogares más vulnerables del país que presenten un o una integrante en situación de dependencia (acorde al Registro Social de Hogares); y (3) el programa no entrega servicios cotidianos de cuidado, sino que sólo ofrece un "respiro al cuidador", lo que implica que para hacerse efectivo ya debe existir un/a cuidador/a principal, a quien el programa le otorga un relevo de algunas horas durante la semana.

Evidentemente, aquí se observan una serie de problemas, que van desde determinantes estructurales que mantienen municipalizado el derecho a la salud, entregando servicios diferenciados a las y los habitantes de las diversas comunas del país; además de que los cuidados de personas en situación de dependencia se conciban como una cuestión relativa a la pobreza, lo que a su vez, sigue reinscribiendo el problema como una cuestión privada, que debe ser resuelta por cada familia $-\mathrm{y}$ especialmente-, por las mujeres de cada familia. De hecho, según el Estudio Nacional de Dependencia en Personas Mayores, realizado por el Servicio Nacional de Adultos Mayores (2010) un 85,6\% de las personas cuidadoras de personas mayores con dependencia son mujeres; y en el caso de discapacidad, acorde a los datos arrojados por el Segundo Estudio Nacional de Discapacidad (ENDISC), realizado por el Servicio Nacional de Discapacidad (2015) un 73,9\% de quienes cuidan a personas con discapacidad, son mujeres, de las cuales el $77,1 \%$ son familiares y/o parientes que residen en el mismo hogar de la persona cuidada, sin recibir remuneración alguna por los cuidados (sólo el 6,4\% declara recibir algún tipo de remuneración).

Debe considerarse que las actividades cotidianas ${ }^{3}$ que caracterizan al trabajo de cuidado de personas en situaciones de dependencia cubren una amplia gama, desde la realización de las actividades que garantizan la subsistencia, como comer o ir al baño, hasta actividades que permiten sostener los vínculos sociales, como aquellas ligadas a la participación social. Particularmente, el hecho de que el trabajo de cuidados implique la realización de actividades básicas, repetitivas y cotidianas implica que las mujeres que asumen este trabajo deban dedicarse en forma exclusiva a éstas, teniendo escasa posibilidad de articular dichas actividades con un trabajo remunerado, dado su carácter inflexible (Carrasquer et al., 1998). En este sentido, la alta demanda de este tipo de trabajo está dada por su cotidianidad y rigidez, que al requerir exclusividad, intensifica las desigualdades de género para las mujeres que lo asumen (Rogero, 2010).

En Chile existen iniciativas ciudadanas no-estatales dedicadas a la visibilización del trabajo de cuidados como un asunto político. Estas iniciativas son en su gran mayoría organizadas y promovidas por mujeres cuidadoras de personas que viven situaciones de dependencia, dentro de las cuales se pueden encontrar "Yo cuido cuidadores" y "Mamá Terapeuta". Estas organizaciones realizaron el año 2018 una encuesta online a cuidadores informales, titulada "Informe 1era Encuesta sobre cuidadores informales" (Fundación Mamá Terapeuta \& Asociación Yo Cuido, 2018) que señala que el 97.7\% de las personas que cuidan informalmente son mujeres, considerando un universo total de 906 encuestas. De este total, el 77\% declaró haber tenido que dejar de trabajar en el mercado remunerado por asumir el rol de cuidadora informal, lo que inmediatamente evidencia el empobrecimiento y precarización laboral que esto implica o puede implicar. Es por ello, que la presente investigación se ha propuesto conocer la cotidianidad del trabajo de cuidados, así como los efectos que ha generado en cuidadoras informales de personas adultas en situaciones de dependencia, desde una lectura feminista.

\section{Aproximaciones Teóricas al Trabajo de Cuidado: Una Perspectiva Feminista}

Intentar dibujar límites conceptuales para el trabajo de cuidados resulta sumamente complejo. Precisamente, porque una de sus características es tener límites difusos, que le desbordan como un acto únicamente corporal o mecánico, en donde se puedan observar acciones concretas y determinadas que se puedan cuantificar (Morini, 2014). Por el contrario, el trabajo de cuidados es intraducible a esta lógica, y puede ser comprendido como trabajo emocional, pues más que producir como resultado un objeto, produce vida y bienestar (Morini, 2014). Según Gonzálvez (2018, p. 200) el trabajo de cuidados es "toda actividad -directa o indirecta- que posibilite el bienestar multidimensional de las personas, facilitando el desarrollo y mantenimiento de la vida diaria",

${ }^{2}$ En Chile, una comuna es la unidad territorial más básica de la división administrativo-territorial de país, que es administrada por una Municipalidad o Municipio, cuyos gobernantes son elegidos a través del sufragio popular de sus habitantes cada 4 años.

${ }^{3}$ Como decisión epistémica, he optado por llamar "actividades cotidianas" a aquel grupo de actividades que desde la tradición anglosajona se han denominado “Actividades de la vida diaria”, en sus derivas "básicas" e "instrumentales”. Esto, por acompañar las críticas de autoras como Galheigo (2003, 2012, 2020), Medeiros (2008) y Palacios (2016), quienes denuncian la fragmentación y despolitización que las corrientes anglo han impreso en la profesión, al dar por asumida como cuestión neutra y ahistórica la relación que las actividades humanas poseen con la vida cotidiana, en la que operan una red de relaciones de poder que producen a su vez, las actividades humanas como su efecto. 
lo que a su vez puede caracterizarse desde tres subtipos de actividades diferenciadas que le componen, que para la economista feminista Pérez (2014) serían: (1) las actividades de cuidados directos, que implican una relación directa entre cuerpos y emociones; (2) las actividades que proveen las precondiciones materiales para el cuidado (como el trabajo doméstico); y (3) las actividades de gestión mental, asociadas al control, evaluación, planificación o supervisión del proceso de cuidados.

Es importante enfatizar que, desde la economía feminista, el cuidado se concibe como una necesidad básica y transversal a la vida, derivada de nuestra vulnerable condición humana que requiere de una serie de acciones continuas para mantener la vida (Arendt, 2003; Nussbaum, 2006; Carrasco, 2017). Así, el cuidado no se liga exclusivamente a personas que requieren algún tipo de apoyo por su edad, condición de salud o que vivencian discapacidad, sino que es una necesidad irrecusable como parte de la condición humana. Esta consideración es fundamental, pues permite desplazar aquella pretensión de autonomía y autovalencia que las relaciones económicas y sociales asumen de un sujeto estereotípico proyectado como universal (Nussbaum, 2006). Así, más que pensar en modo binario que sólo existe dependencia, como un estado patológico por fuera de una cierta norma funcional, y autonomía, como un estado asociado a la normalidad, es preciso considerar que "las personas no somos autónomas o dependientes, sino que nos situamos en diversas posiciones en un continuo de interdependencia” (Pérez, 2006, p.14).

En tanto seres de necesidades, inevitablemente debemos articularnos de forma permanente, tanto con otros sujetos como con la naturaleza, para poder dar respuestas sociales y vinculares a nuestras necesidades fundamentales (Pampliega De Quiroga \& Pichon-Rivière, 1985). El modo en que cada sociedad se articula para dar respuesta a dichas necesidades de cuidados, a través de diversos agentes y mecanismos, corresponde a la organización social del cuidado, que incluye actores como las familias, las comunidades, el Estado y el mercado (Gonzálvez, 2018). En sociedades atravesadas por el neoliberalismo, en donde los Estados se restan cada vez más de su responsabilidad de dar respuesta a las necesidades de cuidados, mediante sistemáticas políticas de ajuste estructural, se ha generado una arquitectura institucional generizada (Comas-D’Argemir, 2017; Gonzálvez, 2018), en donde las mujeres absorben las tensiones y crisis derivadas de un modelo neoliberal que no garantiza condiciones para el cuidado (Fraser, 2016).

Dicha arquitectura generizada, produce de modo permanente las relaciones de género como su efecto, requiriéndolas como parte de la organización económica y socio-sexual que sostiene una cierta cotidianidad (Grandón, 2018). Por ello, resulta interesante ligar el trabajo de cuidados con la vida cotidiana, puesto que ésta puede comprenderse como un espejo de la historia (Heller, 1987), en donde desarrollamos y nos apropiamos de las destrezas que requerimos para operar de forma efectiva en la cotidianidad correspondida a nuestro momento histórico, apropiándonos a la vez de estructuras sociales, económicas y políticas, cuestión que nos permite reproducir un orden socioeconómico dado (Pampliega De Quiroga \& Pichon-Rivière, 1985). Así, en la vida cotidiana manifestamos las relaciones sociales que regulan nuestra vida en nuestra propia época (Pampliega De Quiroga \& Pichon-Rivière, 1985), siendo las relaciones de género un tejido fundamental para sostener y reproducir un modelo económico falo/capitalocéntrico que establece una organización sociosexual que usufructúa permanentemente del trabajo invisibilizado de las mujeres (Cameron \& Gibson-Graham, 2003; Federici, 2018). Por ello, la vida cotidiana puede ser comprendida como una encarnación de la red o estructura de relaciones que sostenemos a través de nuestras prácticas vitales, en donde hemos de reconocer que:

El patriarcado y el capitalismo no son objetos separados de sus productores, su realidad objetiva está sostenida por los seres humanos en su práctica vital, y tienen tanta duración como la práctica vital de los humanos que encarnan esa estructura de relaciones (Izquierdo, 1998, p. 219).

Empero, la vida cotidiana no debe ser comprendida como algo dado que pasivamente introyectamos, pues ésta posee un carácter dinámico y paradojal, en tanto aquellas actividades que realizamos para formar el mundo, a su vez, nos conforman como sujetos (Heller, 1987). Este aspecto formativo de la cotidianidad permite evidenciar cómo ésta constituye el escenario en donde desarrollamos las ocupaciones y/o actividades humanas que, a la vez, crean el mundo, lo que en términos epistemológicos permite desplazar la comprensión de la realidad social como una realidad objetiva y natural, y, más bien, evidencia el carácter paradojal de las ocupaciones humanas, pues son las productoras del mundo que, a la vez, las produce (Rubio \& Sanabria, 2011). Así, “el mundo en que hemos nacido, no existiría sin la actividad humana que lo produjo" (Arendt, 2003, p. 37).

Al posicionar la vida cotidiana como una encarnación y un escenario para la reproducción de estructuras y relaciones sociales, se hace necesario comprenderla junto con la noción de sentido común, pues a través de éste las estructuras y relaciones sociales toman sentido y son asumidas como cuestiones naturales. El examen del sentido común que organiza a la vida cotidiana constituye un acto crítico y político en tanto "se somete a cuestionamiento aquello que antes se daba por descontado, aquello que se suponía conocimiento de sentido común, normalidad” (Izquierdo, 1998, p. 16). Es por ello, que el examen crítico de la vida cotidiana 
puede desnaturalizar lo que se ha pretendido como esencia, para así volverlo una contingencia, vale decir, algo sobre lo que se puede intervenir (Izquierdo, 1998).

Bajo esta óptica, podemos observar que la feminización del trabajo de cuidados —entendida como ocupación generizada (Grandón, 2018), no está dada por una esencia o atributo natural de la feminidad, sino que responde auna construcción histórica contingente, que cristaliza en sí las relaciones de género que producen y reproducen un orden económico cimentado en la desigualdad, a partir de una rígida división sexual del trabajo, en donde los hombres se asocian a lo productivo/mercantil/público y las mujeres a lo reproductivo/doméstico/privado (Pérez, 2006). Las labores propias de la reproducción y el cuidado han sido impuestas desde su naturalización biologicista, tal y como afirma Federici $(2013$, p. 39) al seńalar que "una vez que el trabajo doméstico está totalmente naturalizado y sexualizado, una vez que ha pasado a ser un atributo femenino, todas nosotras como mujeres estamos caracterizadas por ello" En este sentido, el trabajo doméstico y de cuidados, pasa a ser una construcción arquetípica del ser mujer, la cual es reproducida por las estructuras sociales, mediante una serie de dispositivos de socialización (Palacios, 2016) que producen a las ocupaciones de cuidado como una labor feminizada (Vidal et al., 2017). Esto provoca que, al asumir el trabajo no remunerado como un atributo naturalmente femenino, éste no se considere como trabajo, sino como un mandato o un deber-ser femenino atravesado por la responsabilidad moral sobre otros y otras.

Dispuesta de este modo, la vida cotidiana de las mujeres forma parte y sostiene una estructura económico-sexual, la que, al usufructuar gratuitamente del trabajo de cuidados, se reproduce sin tener que garantizar en sus mecanismos de acumulación del capital, la reproducción de la vida ni su sostenibilidad.

\section{Metodología}

La investigación corresponde a una producción cualitativa, puesto que ha enfatizado en la exploración de la experiencia cotidiana del trabajo de cuidados, en donde "los escenarios y las personas son vistos desde una perspectiva holística, es decir, las personas, los escenarios o los grupos no son reducidos a variables, sino considerados como un todo" (Taylor \& Bogdan, 1987, p. 7). Además, se enmarca como una investigación crítica y feminista, reconociendo que como investigadora he dado lectura e interpretación a partir de mi propia situación en el mundo, por lo que en ningún caso mis apreciaciones serán neutras (Guba \& Lincoln, 1994; Haraway, 1995), reforzando nuevamente la elección por los métodos cualitativos, en tanto éstos:

[...] toman la comunicación del investigador con el campo y sus miembros como una parte explícita de la producción de conocimiento, en lugar de excluirla lo más posible como una variable parcialmente responsable. Las subjetividades del investigador $y$ de aquellos a los que se estudia son parte del proceso de investigación (Flick, 2004, p. 20).

Respecto a los resguardos éticos, la investigación consideró la participación voluntaria de 7 mujeres, quienes suscribieron un consentimiento informado tras conocer los objetivos y encuadre teórico de la investigación. Sus identidades aparecen bajo nombre ficticios que ellas misma escogieron, salvo el caso de una que prefirió aparecer con su nombre real, a modo de visibilización política producto de su activismo por la ampliación del derecho al cuidado en Chile.

Como técnicas de producción de información, se realizaron entrevistas semi-estructuradas y observaciones participantes en los contextos cotidianos de cada una de las participantes, entre los meses de octubre del 2018 y abril del 2019 Con relación a la primera técnica, ésta fue seleccionada por su carácter conversacional flexible, que supera la lógica de pregunta-respuesta, y permite profundizar en temáticas emergentes, pese a contar con un guión previo, cuyo orden varía según la conversación con cada entrevistada (Hammer \& Wildavsky, 1990). Como técnica secundaria de producción de información, se realizaron observaciones participantes de la cotidianidad de las cuidadoras participantes, para así "ser testigo de los comportamientos sociales de individuos o grupos en los propios lugares de sus actividades o residencias, sin modificar su marcha ordinaria” (Peretz, 2000, p. 20). Esto permitió observar contextualmente el trabajo de cuidados, siendo parte de las cotidianidades e interacciones sociales de las mujeres participantes, en acciones como desayunar, almorzar o cenar; caminar juntas por sus barrios; acompañarlas en sus labores de cuidados y también apoyarles en la realización del trabajo doméstico asociado al cuidar. Al finalizar cada jornada de observación, de duración estimada entre 2 a 3 horas, se hizo un registro escrito en una bitácora de investigación.

El muestreo se desarrolló de forma intencionada no probabilística, principalmente mediante la estrategia muestreo en cadena o "bola de nieve", en donde "se identifican los casos de interés a partir de alguien que conozca a alguien que puede resultar un buen candidato para participar” (Martínez-Salgado, 2012, p. 616). En este sentido, a partir de redes profesionales y de redes entre las mismas entrevistadas, se fue generando la muestra en cadena, que concluyó en la octava entrevista, a partir del criterio de 
saturación, es decir, el "momento en que la información redunda en lo sabido, y por lo mismo el objeto se ha agotado en sus descriptores” (Canales, 2006, p. 24). Los criterios de inclusión de las participantes fueron los siguientes:

a) Mujer (como identidad de género)

b) Ser cuidadora principal de un adulto/a en situación de dependencia en la ciudad de Santiago de Chile, siendo ésta su actividad principal.

c) Cuidar de manera informal, vale decir, ser parte de una red próxima de la persona cuidada (familiar, vecina, etc.) sin recibir remuneración.

Cabe señalar que, en tanto la investigación buscó conocer la cotidianidad del trabajo de cuidados y sus efectos en las vidas de las cuidadoras, no fue relevante realizar una diferenciación muestral según la condición de salud de la persona cuidada ni el tipo de vínculo sostenido con ella, pues primaron los criterios de inclusión en base a la experiencia de las cuidadoras. De todos modos, estos elementos se han incluido en la siguiente Tabla 1, para evidenciar la diversidad de situaciones de las participantes, que incluyen características como su edad, profesión u oficio, vínculo sostenido con la persona cuidada, tiempo cuidando, condición de salud de la persona cuidada y nivel socioeconómico, además de sus nombres ficticios:

Tabla 1. Caracterización de las mujeres que participaron de la investigación.

\begin{tabular}{|c|c|c|c|c|c|c|}
\hline Nombre & Edad & Profesión u oficio & $\begin{array}{l}\text { Persona a la que } \\
\text { auidan }\end{array}$ & Condición de salud & $\begin{array}{l}\text { Tiempo } \\
\text { auidando } \\
\text { (en ańos) }\end{array}$ & $\begin{array}{c}\text { Nivel } \\
\text { socioeconómico }\end{array}$ \\
\hline Aurora & 65 & Contadora & Esposo (69) & Parkinson espástico & 5 & Medio-alto \\
\hline Mariana & 49 & $\begin{array}{l}\text { Técnica en Educación de } \\
\text { párvulos }\end{array}$ & Mamá (73) & $\begin{array}{l}\text { Alzheimer, daño orgánico producto de } \\
\text { accidente cerebro vascular (ACV) y } \\
\text { demencia. }\end{array}$ & 4 & Bajo \\
\hline Carolina & 55 & $\begin{array}{c}\text { Técnico asistente de } \\
\text { personas adultas mayores }\end{array}$ & Padre (91) & Demencia & 3 & Medio-bajo \\
\hline Fanny & 54 & $\begin{array}{c}\text { Asesora del hogar; } \\
\text { vendedora de chocolatería }\end{array}$ & Hija (25) & Parálisis cerebral & 25 & Baja \\
\hline Victoria & 52 & Ingeniera informática & Hijo (19) & $\begin{array}{l}\text { Trastorno del Espectro Autista (TEA) y } \\
\text { epilepsia refractaria }\end{array}$ & 19 & Medio \\
\hline Ofelia & 58 & $\begin{array}{l}\text { Dueña de casa; vendedora } \\
\text { en ferias libres; costurera. }\end{array}$ & Hijo (38) & $\begin{array}{c}\text { Síndrome de Williams (discapacidad } \\
\text { intelectual) }\end{array}$ & 38 & Bajo \\
\hline Matea & 50 & Ingeniera & Hija (27) & Discapacidad intelectual & 1 & Media \\
\hline
\end{tabular}

Las entrevistas fueron grabadas y transcritas, para luego realizar un análisis de contenido, comprendiendo esta técnica como un modo de tratar los contenidos existentes en los mensajes, con el objetivo de poder interpretarlos (Bardin, 1996). Esto, implica determinar unidades de codificación que logren dar cuenta de los contenidos, ya sea temáticos o de significados, siempre con miras a los contextos que los producen, considerando no sólo lo evidente, sino también aquellos contenidos latentes u "ocultos" en los procesos de comunicación (Bardin, 1996). Para facilitar la aplicación de esta técnica de análisis, se utilizó el programa de análisis de datos cualitativos Atlas.ti 2.0@, a partir del cual se analizó la información en las 2 categorías siguientes:

1. Cotidianidad del trabajo de cuidados

2. Efectos de la asunción del trabajo de cuidados en la vida de las cuidadoras

\section{Resultados}

\section{La cotidianidad del trabajo de cuidados}

A partir de los relatos de las entrevistadas se han podido identificar diversas actividades y tareas que componen la cotidianidad del trabajo de cuidado de personas adultas en situación de dependencia, que exceden solamente a tareas de cuidado directo como alimentar, vestir o bañar. Como se ha señalado anteriormente, según Pérez (2014) estas actividades se pueden clasificar en tres: a) Actividades de cuidados directos; b) Actividades que proveen las precondiciones materiales para el cuidado; y c) Actividades 
de gestión mental. A partir de esta conceptualización ${ }^{4}$, y en base a lo observado, se ha decidido ampliar este último grupo de actividades, pues la investigación mostró que para el caso de los cuidados de personas en situación de dependencia, existe una serie de acciones de gestión que no sólo se asocian a lo "mental”, sino también a lo médico y también a lo institucional.

Sobre el primer grupo de actividades, relativas al cuidado directo — o corpocuidado como he preferido llamar —, son aquellas destinadas a satisfacer las necesidades vitales y orgánicas básicas de las personas cuidadas, e implican una relación corporal, presencial y directa entre la persona cuidadora y la persona cuidada. Al pensar este grupo de actividades desde las claves de Arendt (2003) es posible comprenderlas como actividades de labor, vale decir, actividades que responden a las cíclicas e interminables necesidades orgánicas de los cuerpos, de cuya resolución y respuesta depende la supervivencia y la vida, tal y como expresa una entrevistada al señalar que: "Yo no vivo a través de ella, pero ella sí vive a través de mí, porque si yo no la alimento, yo no la mudo, yo no la baño, si no la levanto, si no me preocupo si está respirando, ella no vive" (Fanny).

Como evidencia la cita, en situaciones de dependencia severa incluso la confirmación de si la persona cuidada está respirando conforma parte de este grupo de actividades de corpocuidado, así como otras actividades identificadas a partir de las entrevistas y observaciones, tales como: dar de comer, bañar, mudar, limpiar y asear, lavar los dientes, peinar, afeitar, vestir/desvestir, administrar medicamentos, acostar/levantar, trasladar, movilizar, apoyar las transiciones de posturas -como por ejemplo, el paso de la posición decúbito supino (recostado/a) hacia sedente y bípedo, o bien las transferencias entre la cama y otras superficies como sillas de ruedas o inodoro. La satisfacción de las necesidades vitales constituye un eje central y fundamental en el trabajo de cuidados de personas adultas en situación de dependencia, y dada su ciclicidad y reiteración, la vida cotidiana de las mujeres que cuidan se ve organizada a partir de la resolución de estas necesidades, que en muchos casos, además conllevan una especialización técnica por los requerimientos propios de cuidar a personas con situaciones de salud complejas. La ejecución de estas tareas, que implica relaciones entre cuerpos de modo directo, hace necesario destacar el lugar que el cuerpo de las cuidadoras ocupa en el trabajo de cuidados. A diferencia de muchos trabajos remunerados cuyo uso del cuerpo representa un esfuerzo leve o moderado, como aquellos dedicados a lo administrativo, el trabajo de cuidados depende directamente de las capacidades corporales de la persona que cuida. Por ejemplo, tener que agacharse para poder asear a la persona cuidada o la gran carga de peso que se debe realizar para transferir a las personas de un sitio a otro, implica el uso y esfuerzo directo del cuerpo, cuestión que, al tratarse de personas adultas, implica una gran carga de peso y un elevado esfuerzo.

Respecto a las actividades indirectas del cuidado, éstas se asocian al trabajo doméstico, como cocinar, ordenar y limpiar, y son aquellas que proveen de las precondiciones materiales para el trabajo de corpocuidado. Todas las participantes reconocieron realizar estas tareas como parte importante de su cotidianidad, muchas veces en forma yuxtapuesta al trabajo de corpocuidado, lo que permite observar que ellas desempeñan en forma paralela dos trabajos no remunerados en un mismo espacio: el trabajo de cuidados y el trabajo doméstico. Como he planteado, resulta complejo intentar separar ambos trabajos, pues las acciones del trabajo doméstico permiten crear las precondiciones materiales para que las actividades de corpocuidado se desarrollen; por ejemplo, la actividad de cocinar permite luego alimentar a la persona cuidada, así como lavar y ordenar la ropa permite vestir. Por ello, se observa que tanto las actividades de corpocuidado, como las actividades indirectas de tipo doméstico están íntimamente relacionadas, cuestión que se hace evidente en las rutinas cotidianas de las mujeres.

Un aspecto relevante de resaltar de este punto es que en varios casos se observó que las actividades indirectas de cuidado no sólo se destinan a crear las condiciones óptimas para el cuidado de la persona en situación de dependencia, sino que éstas además se suelen realizar para núcleos familiares completos, lo que aumenta considerablemente la carga y tiempo de trabajo de las mujeres. Así, en el espacio del hogar se asume que las mujeres cuidadoras deben sobrellevar, además, el trabajo doméstico de todo el hogar, invisibilizando la ejecución paralela de dos trabajos altamente demandantes, lo que produce una densificación del tiempo de las mujeres, esto es, una experiencia del tiempo que está caracterizada por la permanente superposición de una serie de trabajos no remunerados que se realizan en un mismo tiempo-espacio (Durán, 2010). En la yuxtaposición de las actividades de corpocuidado y de cuidado indirecto, se propendió a generar una sobrecarga laboral en las participantes, quienes reconocieron un círculo vicioso de producción de sobrecarga, dado porque en su continua realización de actividades de cuidado, desarrollaban mayores destrezas y habilidades para hacerlo, lo que las familias naturalizaban como de su exclusiva responsabilidad. A su vez, esto generaba en que las cuidadoras asumieran ser las personas más aptas y capaces para la realización de las distintas actividades (como preparar comidas especiales, ajustar catres clínicos y ayudas técnicas, organizar la medicación, etc.), sintiéndose imprescindibles e irremplazables en

${ }^{4}$ Es importante seńalar que la presente investigación no pretende instalar una nueva forma de organizar o fragmentar las actividades cotidianas como lo han hecho las tradiciones anglosajonas de la profesión, sino que busca visibilizar la complejidad de aquellas acciones que, de otro modo, quedarían ocultas y naturalizadas bajo la concepción unívoca y androcéntrica de trabajo, que le considera únicamente como actividad remunerada. 
la escena del cuidado. Este círculo vicioso se vio reforzado por la tecnificación del rol de cuidados de las mujeres, quienes han debido incorporar una serie de técnicas y maniobras específicas, algunas de alta complejidad, lo que las ha vuelto expertas por experiencia.

Por otra parte, a partir de la experiencia de las participantes, se observó que como parte del trabajo cotidiano de cuidados existen actividades de gestión mental, gestión médica y gestión institucional. La primera de ellas, gestión mental, puede ser comprendida como un modo de estar-en-el-mundo asociado a la alerta permanente, a partir de la necesidad de las cuidadoras de estar constantemente dispuestas ante cualquier necesidad o urgencia de la persona cuidada. La gestión mental se pudo apreciar como una serie de operaciones mentales asociadas a la conexión permanente con las personas cuidadas y sus horarios y/o rutinas, en tanto conlleva acciones de control, evaluación, planificación y supervisión del proceso de cuidado (Pérez, 2014). Se observó que este tipo de gestión se ejecuta de manera permanente por las cuidadoras, quienes pese a no estar de manera directa asumiendo la responsabilidad del cuidado por estar en otro espacio realizando otra actividad como un trámite o una salida social, reconocieron estar permanentemente conectadas con las rutinas cotidianas de cuidado, lo que las mantenía inquietas, impidiendo su desconexión del trabajo de cuidados. Esta inquietud o imposibilidad de desconectarse, condujo a muchas participantes a abandonar sus actividades sociales o incluso proyectos de vacaciones, por asumir que estarían constantemente atentas al trabajo de cuidados y no podrían disfrutar efectivamente de esos espacios, como señaló una participante:

Yo a veces digo, me gustaría irme un fin de semana a la playita, sola, y después digo chuta y voy a estar allá y la Estela ¿Habrá tomado once? ¿Le habrán dado almuerzo? ¿Habrá comido bien? ¿La habrán mudado? ¿Se habrá pasado? ¿Le habrán cambiado la ropa de cama? Entonces, no. No po, ¿para qué voy a salir? (Fanny).

Muy ligadas a este modo de estar en el mundo, se encuentran las actividades que he denominado gestión médica, categoría referida a aquellas actividades destinadas de manera específica al control, supervisión y acompañamiento de procesos de salud-enfermedad y rehabilitación. Esto, se expresó en tareas concretas de alta especialización como solicitud de citas y exámenes médicos, calendarización estratégica de controles médicos recurrentes y de especialidad, dosificación y cumplimiento de horarios de toma de medicamentos, acompañamiento a realización de exámenes y retiro de sus resultados, aprendizaje y ejecución de ejercicios de rehabilitación como los kinesiológicos, fonoaudiológicos o de terapia ocupacional, etc. Para llevar a cabo este tipo de gestión, las participantes señalaron usar diversas estrategias, como poner alarmas diarias en sus celulares para recordar los horarios de los medicamentos, calendarizar las intervenciones médicas en pizarras visibles en espacios comunes, usar agendas o cuadernos, además de familiarizarse con nomenclaturas médicas. Un ejemplo que entrecruza tanto la gestión mental como la gestión médica es el que se expone a continuación:

A veces ponte tú, el papá se queda con él. Él "ya" me dice, "sale, no te preocupes, yo me quedo aquí con él". $Y$ claro, yo salgo, pero uno igual está: que a esta hora los remedios, yo lo llamo por... no se te olvide, a esta hora los remedios, ¿cachai? (Victoria).

Muchas de las entrevistadas, especialmente aquellas asociadas a niveles socioeconómicos medios y bajos, describieron en su vida cotidiana un tercer tipo de gestión, que he denominado gestión institucional. Ésta alude a todas las acciones realizadas para favorecer el ejercicio efectivo a derechos sociales de las personas cuidadas, como el derecho a la salud, a la vivienda o a la protección social. Algunas entrevistadas reconocieron que para poder conquistar en forma efectiva estos derechos debían acudir de forma sostenida y reiterada a distintas entidades de administración estatal y/o municipal como consultorios, municipios y hospitales, en una relación de tensión con los mismos, para poder contar con insumos clínicos o bonos de dinero. Varias entrevistadas reconocieron que estos dispositivos suelen proceder de forma burocrática y malos tratos, ante lo que ellas han tenido que realizar reclamos, exigiendo sus derechos y los de las personas que cuidan, ya sea llamando por teléfono, enviando cartas, acreditando diagnósticos y gastos económicos en pos de recibir subvenciones y beneficios, como se reconoce en el siguiente relato:

Si yo con decirte que yo, yo a veces no le tenía pañales a mi hija, ni pañales. No importa decía yo. Y todos me decía ;ay! pero es que anda a la municipalidad, cuenta tu caso y en tu caso salías destrozada, porque la asistente te humillaba, y por qué no se cuidó señora, y quién la mandó a tener tantos críos (Fanny).

Como se evidencia en la cita, además de una acción de mendigar derechos sociales, la cuidadora fue objeto de una moralización sexual sobre su vida, a partir de una jerarquía de poder institucional. Resulta interesante enfatizar que este tipo de gestión se observó especialmente en aquellas cuidadoras pertenecientes a estratos socioeconómicos más bajos quienes, al no poder acceder en forma privada a ciertos servicios, dependían exclusivamente de la provisión estatal y municipal. Considerando la profundización neoliberal de Chile, y la ausencia de políticas públicas que trasciendan el enfoque de necesidades para operar 
desde un enfoque de derechos y de género, la gestión institucional puede pensarse a la luz del concepto interseccionalidad, el cual acentúa cómo la red de relaciones sociales -como las de clase, raza o diversidad funcional, son consubstanciales y coextensivas con las relaciones de género, intensificando sus opresiones y efectos de dominación, en tanto estas relaciones se construyen recíprocamente (Viveros, 2016). Así, la gestión institucional se expresa como un cuerpo de actividades necesarias de asumir por mujeres cuidadoras cuya clase social no les permite acceder a servicios básicos de forma extra-estatal (privada), cuestión paradojal pues, pese a tratarse de derechos sociales básicos como la salud o la vivienda, éstos deben ser activamente gestionados por ellas, volviéndose parte cotidiana de sus actividades de cuidado.

Finalmente, es necesario señalar cómo todas estas actividades cotidianas del trabajo de cuidados guardan relación con una cierta temporalidad que las sujeta. La organización del tiempo de vida de las mujeres que cuidan está siempre atravesada por las actividades de cuidado, pero también por las demandas potenciales de cuidado -que pueden o no concretarse-, cuestión que dificulta su libre disposición y uso del tiempo. En este sentido, ellas están "habitualmente están faltas de tiempo, porque son donantes de tiempo" (Carrasco, 2006, p. 54), lo que genera que constantemente deban estar realizando una serie de gestiones mediadas por terceras personas para poder organizar sus tiempos cotidianos. En los relatos de las entrevistadas fue posible identificar cómo sus rutinas temporales se estructuraban en base al tiempo orgánico de la persona cuidada, fenómeno que contribuye a una indistinción del tiempo de trabajo y tiempo de vida (Durán, 2010; Morini, 2014), pues en tanto las necesidades orgánicas no cesan, asimismo, el trabajo de cuidados no tiene límites de inicio ni de fin, sino que más bien constituye una continuidad temporal.

\section{Efectos del trabajo de cuidado en la vida cotidiana de las mujeres que cuidan}

Como se ha evidenciado, el trabajo de cuidados de personas adultas en situación de dependencia es sumamente demandante y posee escasos márgenes de flexibilidad, en tanto su temporalidad asociada a las necesidades orgánicas de la persona cuidada le otorgan un carácter cíclico y reiterativo. Además, tiene como característica el encontrarse territorializado al espacio del cuidado la casa-, lo que restringe las posibilidades de escisión de la escena del cuidado por las mujeres. Todo lo anterior, ha generado en las participantes una serie de efectos múltiples, que incluyen efectos en su salud física y mental, en sus relaciones sociales y espacios de participación, así como también efectos económicos que las han empobrecido y vuelto económicamente dependientes de otros y otras.

Respecto a los efectos personales, se identificó que la mayoría de las mujeres experimentaron lesiones y/o dolencias físicas producto de la sobrecarga del trabajo de cuidados, expresada en hernias lumbares, dolores musculares y/o articulares y síndrome de colon irritable. Al no tener relevos del cuidado, las participantes refirieron la dificultad de poder sanar dichas lesiones, ya fuese por la dificultad para asistir a centros de salud, por la imposibilidad de dar descanso a sus músculos y articulaciones, o bien, por impedimentos económicos para realizar tratamientos médicos. Además de las lesiones y dolencias físicas, se observó que gran parte de las mujeres entrevistadas experimentaban problemas de salud mental, asociados al agotamiento, estrés, angustia o miedo. Las participantes refirieron no poder enfermarse, producto de ser imprescindibles en la escena del cuidado, como evidencian los siguientes relatos:

Aparte de estrés, frustración, jay! ' Decepción, grrrrr! ïAngustia!! (llora) Tristeza a veces (llora) y todo eso te lleva a la depresión, y yo no me puedo deprimir tampoco (Matea).

Yo me exijo mucho y me angustio y se me pone aqui el pecho se me aprieta, y actú, y de repente actú, y al actuar no hago bien las cosas, tuve un periodo en que se me olvidaron, se me han estado olvidando todo, y dije no, yo tengo alzheimer, yo tengo demencia, yo tengo algo, porque todo, dejaba la tetera prendida, se me olvidaba las fechas de los controles, de los medicamentos, estuve así como en una nebulosa, así como que andaba así, así (Carolina).

Se observó que estos efectos sobre la salud de las cuidadoras estaban asociados, además de la sobrecarga, a la postergación del cuidado propio dada por la imposibilidad de cuidar-se. Todas las entrevistadas reconocieron lo difícil que les resultaba poder realizar actividades de autocuidado de su salud en medio de la ejecución del trabajo de cuidados, el cual como he señalado, las mantiene territorializadas o circunscritas al espacio de reproducción de la vida -la casa, con escasas posibilidades de apoyos y relevos en el rol de cuidadora.

Otro efecto cotidiano muy común entre las participantes fue el mal dormir y la escasez de tiempos de descanso. En algunos casos, el mal dormir resultaba como producto de las complejidades propias de las condiciones de salud de las personas cuidadas, como apneas, trastornos del sueño u otras; mientras que, en otros casos, el mal dormir se asociaba a un estado de alerta permanente 
que les impedía tener un descanso pleno. Sólo en algunos casos, se observó que las mujeres que poseían mayores redes de apoyo podían lograr gestionar tiempos y espacios para el descanso, aunque éstos en su mayoría se realizaban dentro del mismo espacio en donde estaba la persona cuidada, cuestión que les dificultaba la desconexión total respecto de las rutinas y responsabilidades del cuidado. Aparece aquí un fenómeno interesante, y es que incluso para poder descansar las mujeres debían realizar gestiones y coordinaciones con sus redes próximas, lo que supeditaba sus posibilidades de descanso a la disponibilidad de una tercera persona que lo hiciese efectivo.

Además, dada la convivencia con la persona cuidada, las participantes declararon tener una pérdida de privacidad en algunas actividades cotidianas básicas, como ir al baño a realizar sus necesidades o en el desarrollo de su vida sexual. Para varias participantes la posibilidad de sostener una relación sexo-afectiva siendo cuidadora de una persona en situación de dependencia era compleja de concebir, dadas las limitantes de espacio y de tiempo, como se evidencia en los siguientes relatos:

Por ejemplo ahora, si yo quisiera tener un pololo ${ }^{5}$, cómo lo traigo pa acá, y si mi papi tiene una emergencia y se mandó una cagá, no sé po, te digo, dónde, cómo, no puedo, estoy así como... bien básico (Carolina).

Te pongo el ejemplo de que mi pareja un día me decía quiero salir contigo, o quiero que vayamos a tal parte, ¿Con quién dejaba a mi hijo? Mis hijas ya tienen su vida, ella tiene su hija y su pareja, ella sale con su pareja y yo no podría molestar (Ofelia).

Para muchas cuidadoras, la posibilidad de proyectar una relación de pareja era nula, y la asumían como algo caduco. Por otra parte, dentro de los principales efectos a nivel familiar, se observó que al momento de decidir quién asumiría el trabajo de cuidados, las familias se reorganizaron siguiendo una rígida división sexual del trabajo reproductivo, siendo en todos los casos las mujeres las cuidadoras. En esta decisión sexista, primó el vínculo que las mujeres sostenían con la persona que presentaba dependencia — como el de esposa, hija o madre-, cuestión que al darse entre familias compuestas sólo por mujeres, se reconsideraba en función del nivel de ingresos. Así, las mujeres que percibían menores salarios o que tenían condiciones laborales inestables, eran quienes preferentemente asumieron el trabajo de cuidados. Además, en muchos casos, las mujeres realizaban de manera yuxtapuesta una serie de otros trabajos no remunerados de cuidado, especialmente cuando existían nietos o nietas en los hogares. Se reconoció también que la experiencia del cuidado posee grandes diferencias según el tipo de vínculo que las mujeres sostienen con la persona cuidada, puesto que en el caso de las cuidadoras-madres, el trabajo de cuidados iniciaba al mismo tiempo en que nacían sus hijos e hijas, lo que volvía al cuidado su única proyección ocupacional, incluso por varias décadas. Además, en estos casos, los esencialismos de la maternidad hacían aún más automática la decisión de que fuesen ellas quienes cuidasen, quedando a temprana edad privadas de acceder a niveles superiores de educación, a trabajos remunerados, a ocio y tiempo libre, así como a diversas elecciones ocupacionales y derechos sociales.

También, se observaron una serie de estragos económicos para las participantes, quienes tras asumir el trabajo de cuidados, debieron abandonar sus trabajos remunerados, salvo aquellas que se desempeñaban como "dueñas de casa", a quienes se les intensificó notablemente la carga global de trabajo. Esto implicó sin excepción una fuerte precarización expresada en la ausencia de remuneración, irregularidad o suspensión de pagos de seguros de salud y fondos previsionales de jubilación, existencia de "lagunas" laborales en los currículums de las cuidadoras con carrera profesional, y dependencia económica de terceras personas y/o entidades, volviéndose así personas económicamente dependientes de sus familias o bien, de beneficios estatales como bonos o pensiones. Resulta interesante destacar que el hecho de que todas las participantes hayan debido abandonar el trabajo remunerado para dedicarse al cuidado, pone de manifiesto el vacío de cuidados existente en la vida social (Fraser, 2016), en donde la organización social del cuidado recae por completo en las mujeres. Así, para poder cuidar, son las mujeres las que "están dejando de estudiar, trabajar en el mercado, están perdiendo años de aportes para su jubilación del futuro y posibilidades de desarrollo y realización personal" (D’Alessandro, 2018, p. 161).

Por contrapartida, en todos los casos se evidenció que el cuidado en situaciones de dependencia representa un elevado gasto económico, cuestión paradojal con el hecho de que este trabajo no sea remunerado, pese a su alta especialización y demanda. Como consecuencia, las mujeres han debido desarrollar estrategias de autogestión laboral, tanto para cubrir su subsistencia, así como para poder mejorar parcialmente sus condiciones de vida, que se observan precarizadas. Por ello, además del cuidado y de la serie de trabajos no remunerados que se yuxtaponen en su cotidianidad, las mujeres también han debido sobrellevar iniciativas de autogestión laboral que puedan compatibilizarse con el trabajo de cuidados, destacándose actividades como chocolatería, costurería o trabajos online (informáticos).

${ }^{5}$ Chilenismo que significa "novio" o "pareja". 
Finalmente, es preciso señalar que las mujeres que cuidan informalmente a personas en situación de dependencia se transforman en cuidadoras de tiempo completo, desplazando sus identidades ocupacionales hacia la identidad de cuidadora, como cuestión que organiza su vida cotidiana y que, para algunas participantes, se transformó en una identidad política. Del total de participantes, la mitad declaró participar activamente de redes de organización de cuidadoras, como parte de una lucha política en pos del reconocimiento de este trabajo invisible por el Estado y la sociedad civil, a partir del reconocimiento del cuidado como un derecho social. En tiempos prácticamente inexistentes, las mujeres logran articularse y organizarse políticamente, para en ello, sustituir los estragos de la profundización neoliberal en las políticas públicas y en la precarización de los vínculos humanos, principalmente a través de redes sociales como Facebook, Instagram y WhatsApp.

\section{Discusión}

A partir de lo investigado, pudo reconocerse cómo las vidas cotidianas de las mujeres que cuidan informalmente a personas adultas en situación de dependencia constituyen un espejo de la historia (Heller, 1987), que expresa la voracidad y el usufructo que el sistema socioeconómico actual realiza sobre los trabajos no remunerados asumidos por las mujeres. Al ser un trabajo que se desarrolla en el mismo tiempo/espacio en que transcurren sus vidas cotidianas, —a diferencia de la mayoría de los trabajos remunerados que se realizan fuera del hogar, con una jornada delimitada de inicio y fin-, esta característica de convivencia de las cuidadoras con la persona cuidada propendió a territorializar con mayor fuerza a las mujeres hacia los espacios domésticos. Esto, además de la gran sobrecarga propia de las múltiples acciones que componen al trabajo de cuidados, tuvo efectos multidimensionales en sus vidas cotidianas, como el abandono de sus trabajos remunerados, cuestión que las volvió económicamente dependientes; limitación para participar en espacios sociales y actividades significativas; impactos negativos en su salud física y mental, disminución casi completa de tiempos de descanso y ocio, e incompatibilidad del trabajo de cuidados con el desarrollo de una vida sexo-afectiva satisfactoria o estable. Con esto a la base, se puede afirmar que las participantes de la investigación se han visto precarizadas, no sólo como cuestión económica, sino como parte de una experiencia vital cotidiana, pues al ser dependientes económicamente de otros y otras, han perdido posibilidades de ejercer su autonomía, tanto en relación al libre uso y disposición de su tiempo, así como en la determinación de sus actividades y sus finanzas.

Pese a los cambios contemporáneos, en donde cada vez más las mujeres participamos en los mercados laborales remunerados, la división sexual del trabajo aún permanece como un resabio rígido del "teatro familiar de las relaciones de género" (Segato, 2014, p. 92), forma de socialización que ha producido al sujeto-mujer como un sujeto esencialmente dador de cuidados, por lo que ante alguna condición de salud que genere dependencia, todas las mujeres aparecemos como potenciales cuidadoras, en tanto es condición de la feminidad. Resulta importante recalcar que además de este componente de socialización y subjetivación, la reproducción de las relaciones de género encuentra condiciones materiales y objetivas que facilitan su reinscripción en la vida cotidiana, pues en tanto existan vacíos del cuidado producto de la sexista organización social del cuidado, disminución de la inversión estatal en políticas de bienestar y brechas salariales que desincentiven la permanencia de las mujeres en los mercados laborales remunerados, el fenómeno de quién cuida ante una situación de dependencia, seguirá siendo un asunto feminizado. Este análisis permite sostener cómo las ocupaciones y actividades humanas constituyen una expresión social y cultural —más que un impulso intrínseco e individual-, construidos en una compleja red de relaciones de poder que se interseccionan. Así, podemos entender al trabajo de cuidados como una ocupación generizada cuya reproducción encuentra lugar en las estructuras y aparatos del Estado, en la organización de la economía, en los procesos de socialización, en las representaciones culturales, en los sentidos comunes, esto es, en la compleja textura de la cotidianidad (Galheigo, 2020).

Ante ello, la organización política de las mujeres que cuidan constituyó un hallazgo político muy importante, pues desafía modos unívocos para pensar alternativas y soluciones a las crisis del cuidado, siendo así no solamente el Estado la única vía a la cual apelar, en tanto las mujeres y su capacidad de agencia, ponen de manifiesto la posibilidad de realizar prácticas de resistencia allí donde operan las relaciones de poder (Foucault, 1980). Asimismo, este hallazgo evidencia cómo las mujeres se desplazan de la representación de un sujeto-víctima, abandonando en ello el "espectáculo de la subordinación” (Segato, 2014, p. 99) para afirmar en sus resistencias colectivas su capacidad de autodeterminación, a la vez que construyen una identidad política colectiva, lo que puede leerse también a la luz del concepto de ocupaciones colectivas (Ramugondo \& Kronenberg, 2015; Palacios, 2017). Éstas, surgen intencionadamente como una propuesta crítica de organización ante un malestar común, que para este caso, deriva de una inequitativa y sexista organización social del cuidado. 


\section{Condusiones}

Ante la retirada de los Estados neoliberales de los asuntos de bienestar social, la creciente mercantilización del cuidado y las reorganizaciones de las estructuras familiares, son las mujeres quienes absorbemos las tensiones de un modelo socioeconómico que no considera el cuidado como condición básica de la organización social, especialmente cuando se refiere a personas adultas en situaciones de dependencia. Visto de este modo, si bien esta investigación es específica en ahondar sobre la relación entre género, cuidados y dependencia desde una perspectiva feminista, aun así, resultan necesarias futuras investigaciones que profundicen y complejicen el análisis, intencionando otras relaciones a las ya expuestas en pos de pensar interseccionalmente este problema, con variables como la clase social; condición de salud de la persona cuidada; tipo de vínculo entre cuidadora y persona cuidada; migración y cadenas globales del cuidado, y organización política de cuidadoras.

Finalmente, es preciso señalar que, como investigadoras feministas, tenemos la necesidad de feminizar las investigaciones sociales, iluminando aquellos problemas que se han considerado como "de segundo orden" o como "problemas de las mujeres", evidenciando que lo personal es político, y que las ocupaciones y actividades humanas se producen en una compleja trama de relaciones de poder. La posibilidad de iluminar einvestigar aquellos territorios que históricamente hemos ocupado como sujetos femeninos, constituye una necesidad y una deuda que las mujeres tenemos con nosotras mismas, en pos de evidenciar la arquitectura política que ha sostenido a aquellas ocupaciones feminizadas usufructuadas por el capital.

\section{Agradecimientos}

Agradezco a la Agencia Nacional de Investigación y Desarrollo (ANID, Chile), por el financiamiento de mis actuales estudios doctorales a través de la beca PFCHA/Doctorado Nacional/2020-21201656. Agradezco a cada una de las participantes de esta investigación por confiar en el valor ético y político de esta propuesta. También, a mi maestra Ximena Azúa Ríos, por su guía y apoyo en la investigación; y a Pilar Catalán Meneses, por sus atentos comentarios en la revisión final de este texto.

\section{Referencias}

Arendt, H. (2003). La Condición Humana. Buenos Aires: Paidós.

Arriagada, I. (2004). Dimensiones de la pobreza y políticas desde una perspectiva de género. In Anales de la Reunión de Expertos. Políticasy programas de superación de la pobreza desde la perspectiva de la gobernabilidad democrática y el género. Quito: CEPAL.

Arriagada, I., \& Todaro, R. (2012). Cadenas Globales de Cuidado: El papel de las migrantes peruanas en la provisión de cuidados en Chile. Santo Domingo: ONU Mujeres.

Bardin, L. (1996). Análisis de contenido. Madrid: Akal.

Batthyány, K. (2015). Las políticas y el cuidado en América Latina: Una mirada a las experiencias regionales. Santiago: Cooperación Española. Naciones Unidas.

Cameron, J., \& Gibson-Graham, J. K. (2003). Feminising the Economy: Metaphors, strategies, politics. Gender, Place and Culture, 10(2), 145-157. http://dx.doi.org/10.1080/0966369032000079569.

Canales, C. M. (2006). Metodologias de investigación social: Introducción a los oficios. Santiago: LOM.

Carrasco, C. (2006). La paradoja de cuidado: necesario pero invisible. Revista de Economía Crítica, (5), 38-64.

Carrasco, C. (2017). La economía feminista: Un recorrido a través del concepto de reproducción. Ekonomiaz: Revista Vasca de Economía, (91), $52-77$.

Carrasquer, P., Torns, T., Tejero, E., \& Romero, A. (1998). El trabajo reproductivo. Papers: Revista de Sociologia, 55, 95-114. http://dx.doi.org/10.5565/rev/papers. 1934.

Comas-D’Argemir, D. (2017). El don y la reciprocidad tienen género: las bases morales de los cuidados. Quaderns-e de l'Institut Català d'Antropologia, 22(2), 17-32.

Comisión Económica para América Latina y el Caribe - CEPAL. (2011). Informe de la undécima conferencia regional sobre la mujer de América Latina y el Caribe. In Anales de la $11^{\circ}$ Conferencia regional sobre la mujer de América Latina y El Caribe. Brasilia: CEPAL.

Comisión Económica para América Latina y el Caribe - CEPAL. (2017). Informe de la decimotercera Conferencia Regional Sobre la Mujer de América Latina y el Caribe. In Anales de la $13^{a}$ Conferencia regional sobre la mujer de América Latina y el Caribe. Montevideo: CEPAL.

Cuida, C. (2020). Sistema de Apoyos y Cuidados. Conócenos. Recuperado el 03 de septiembre de 2020, de https://www.chilecuida.gob.cl/conocenos/

D’Alessandro, M. (2018). Economía feminista. México: Penguin Random House. 
Durán, M. A. (2010). Tiempo de vida y tiempo de trabajo. Bilbão: Fundación BBVA.

Durán, M. A. (2011). El trabajo del cuidado en América Latina y España (Documento de trabajo número 54). Madrid: Fundación Carolina.

Federici, S. (2013). Revolución en punto cero. Trabajo doméstico, reproducción y luchas feministas. Madrid: Traficantes de sueños.

Federici, S. (2018). El patriarcado del salario: críticas feministas al marxismo. Buenos Aires: Tinta Limón.

Flick, U. (2004). Introducción a la Investigación Cualitativa. Madrid: Morata S.L.

Foucault, M. (1980). Poderes y estrategias. En M. Foucault, Microfisica del poder (pp. 163-174). Madrid: La piqueta.

Fraser, N. (2016). Las contradicciones del capital y los cuidados. New Left Review, 100, 111-132. Recuperado el 15 de septiembre de 2020, de https://newleftreview.es/issues/100/articles/nancy-fraser-el-capital-y-los-cuidados.pdf

Fundación Mamá Terapeuta, \& Asociación Yo Cuido. (2018). Informe 1era Encuesta sobre Cuidadores Informales: la mirada de quienes cuidan en Chile. Chile: Organizaciones Sociales. Recuperado el 15 de septiembre de 2020, de https://docer.com.ar/doc/8x8e00.

Galheigo, S. M. (2003). O cotidiano na terapia ocupacional: cultura, subjetividade e contexto histórico-social. Revista de Terapia Ocupacional da Universidade de São Paulo, 14(3), 104-109. http://dx.doi.org/10.11606/issn.2238-6149.v14i3p104-109.

Galheigo, S. M. (2012). Perspectiva crítica y compleja de la terapia ocupacional: actividad, cotidiano, diversidad, justicia social y compromiso ético-político. Revista Terapia Ocupacional Galicia, 9(5), 176-187.

Galheigo, S. M. (2020). Terapia ocupacional, cotidiano e a tessitura da vida: aportes teórico-conceituais para a construção de perspectivas críticas e emancipatórias. Cadernos Brasileiros de Terapia Ocupacional, 28(1), 5-25. http://dx.doi.org/10.4322/2526-8910.ctoAO2590.

Gonzálvez, H. (2018). Género, cuidados y vejez: Mujeres «en el medio» del trabajo remunerado y del trabajo de cuidado en Santiago de Chile. Prisma Social-Revista de Ciencias Sociales, (21), 194-218.

Grandón, D. (2018). Función económica de las ocupaciones feminizadas no remuneradas: una crítica desde la economía feminista. Revista Ocupación Humana, 18(2), 54-67. http://dx.doi.org/10.25214/25907816.228.

Guba, E., \& Lincoln, Y. (1994) Competing paradigms in qualitative research. In N. Denzin \& Y. Lincoln (Eds.), Handbook of qualitative research (pp. 105-117). London: Sage.

Hammer, D., \& Wildavsky, A. (1990). La entrevista semi-estructurada de final abierto: aproximación a una guía operativa. Historia y Fuente Oral, (4), 23-61. http://dx.doi.org/10.2307/27753290.

Haraway, D. J. (1995). Conocimientos situados: la cuestión científica en el feminismo y el privilegio de la perspectiva parcial. In D.J., Haraway (Ed.), Ciencia, cyborgs y mujeres (pp. 313- 346) Madrid: Ediciones Cátedra.

Heller, A. (1987). Sociología de la vida cotidiana. Barcelona: Península.

Instituto Nacional de Estadísticas de Chile - INE. (2016). Documento de principales resultados ENUT 2015. Santiago de Chile: Departamento de Estudios Sociales. Recuperado en 14 de septiembre de 2020, de https://www.ine.cl/estadisticas/sociales/genero/uso-del-tiempo

Izquierdo, M. (1998). El malestar en la desigualdad. Madrid: Cátedra.

Martínez-Salgado, C. (2012). El muestreo en investigación cualitativa: principios básicos y algunas controversias. Ciencia \& Saude Coletiva, 17(3), 613-619. http://dx.doi.org/10.1590/S1413-81232012000300006.

Medeiros, M. H. R. (2008). Terapia Ocupacional: un enfoque epistemológico y social. Santa Fe: Ediciones Universidad Nacional del Litoral.

Morini, C. (2014). Por amor o a la fuerza: Feminización del trabajo y biopolitica del cuerpo. Madrid: Traficantes de sueños.

Nussbaum, M. (2006). Poverty and Human Functioning: Capabilities as Fundamental Entitlements. In D. B. Grusky \& R. Kanbur (Eds.), Poverty and Inequality (pp.47-75). California: Standford University Press.

Palacios, M. (2016). Conceptualizaciones sobre cultura, socialización, vida cotidiana y ocupación: reflexiones desde espacios formativos. Revista Ocupación Humana, 16(1), 56-69. http://dx.doi.org/10.25214/25907816.9.

Palacios, M. (2017). Acerca de Sentido de Comunidad, Ocupaciones Colectivas y Bienestar/Malestar Psicosocial: Con jóvenes transgresores de territorios Populares (Tese de doutorado). Universidad de VIC, Espanha.

Pampliega De Quiroga, A., \& Pichon-Rivière, E. (1985). Psicología da vida cotidiana. Buenos Aires: Nueva Visión

Peretz, H. (2000). Los métodos en sociologia: la observación. Quito: Abya-yala.

Pérez, A. (2006). Amenaza tormenta: la crisis de los cuidados y la reorganización del sistema económico. Revista de Economía Crítica, (5), 7-37.

Pérez, A. (2014). Subversión feminista de la economía: Aportes para un debate sobre el conflicto capital-vida. Madrid: Traficantes de sueños.

Ramugondo, E. L., \& Kronenberg, F. (2015). Explaining collective occupations from a human relations perspective: bridging the individualcollective dichotomy. Journal of Occupational Science, 22(1), 3-16. http://dx.doi.org/10.1080/14427591.2013.781920.

Rogero, J. (2010). Los tiempos del cuidado. El impacto de la dependencia de los mayores en la vida cotidiana de sus cuidadores. Madrid: IMSERSO.

Rubio, S., \& Sanabria, L. (2011). Ocupación como proceso subjetivante. In C. Rojas (Ed.) Ocupación: sentido, realización y libertad. Diálogos ocupacionales entorno al sujeto, la sociedad y el medio ambiente (pp. 71-105). Bogotá: Universidad Nacional de Colombia. 
Segato, R. L. (2014). La guerra contra las mujeres. Madrid: Traficantes de sueños.

Servicio Nacional de Adultos Mayores - SENAMA. (2010). Estudio Nacional de la Dependencia en Personas Mayores. Recuperado en 18 de Julio de 2020, de http://www.superacionpobreza.cl/wpcontent/uploads/2014/01/estudiodependencia.pdf

Servicio Nacional de Discapacidad - SENADIS. (2015). Segundo Estudio Nacional de la Discapacidad. Santiado: Feyser.

Taylor, S., \& Bogdan, R. (1987). Introducción a los métodos cualitativos de investigación: la búsqueda de significados. Buenos Aires: Paidós.

Vidal, M. I., López, E., \& Royo, N. (2017). Visibilizando los cuidados desde una perspectiva feminista en terapia ocupacional. Revista Terapia Ocupacional Galicia, 15(27), 185-190.

Viveros, M. (2016). La interseccionalidad: una aproximación situada a la dominación. Debate Feminista, 52, 1-17. http://dx.doi.org/10.1016/j.df.2016.09.005.

\section{Autor para la correspondencia}

Débora Grandón Valenzuela

e-mail: dlunagrandon@gmail.com

\section{Editor de Seçáo}

Prof. Dr. Rodolfo Morrison 